Abstract P186 Table 1 Comparison between symptomatic and asymptomatic groups from EBUS culture positive subcohort

\begin{tabular}{|c|c|c|c|c|}
\hline & & $\frac{\text { Symptomatic }}{(\mathrm{n}=19)}$ & $\frac{\text { Asymptomatic }}{(\mathrm{n}=8)}$ & P-value \\
\hline \multicolumn{2}{|c|}{ Gender (M:F) } & $\begin{array}{l}68.4 \%: \\
31.6 \%\end{array}$ & $50 \%: 50 \%$ & 0.42 \\
\hline \multicolumn{2}{|c|}{ Age (mean $\pm S D$ ) (years) } & $35.58 \pm 12.81$ & $46.0 \pm 17.97$ & 0.12 \\
\hline \multirow{3}{*}{ Ethnicity } & White & $15.79 \%$ & $25 \%$ & \multirow{3}{*}{0.81} \\
\hline & Black-African & $26.32 \%$ & $37.5 \%$ & \\
\hline & Indian & $31.58 \%$ & $25 \%$ & \\
\hline \multirow{3}{*}{ Born in UK } & $\mathrm{Y}$ & $5.26 \%$ & $12.5 \%$ & \multirow{3}{*}{0.53} \\
\hline & $\mathrm{N}$ & $89.47 \%$ & $87.5 \%$ & \\
\hline & $\mathrm{U}$ & $5.26 \%$ & $0 \%$ & \\
\hline Duration in & K (median; IQR) & $12(4-19.5)$ & $2(1-6)$ & $0.01^{*}$ \\
\hline \multirow{5}{*}{ Exposures } & Diabetes & $0 \%$ & $14.29 \%$ & 0.33 \\
\hline & HIV & $5.26 \%$ & $0 \%$ & 0.75 \\
\hline & Travel endemic & $68.42 \%$ & $42.86 \%$ & 0.23 \\
\hline & Smoking & $13.33 \%$ & $14.29 \%$ & 1.00 \\
\hline & TB contact & $37.5 \%$ & $33.33 \%$ & 1.00 \\
\hline \multirow{5}{*}{$\begin{array}{l}\text { Disease } \\
\text { sites }\end{array}$} & Pulmonary & $47.37 \%$ & $0 \%$ & \multirow{5}{*}{$0.01^{*}$} \\
\hline & Pleural & $5.26 \%$ & $0 \%$ & \\
\hline & ETLN & $26.32 \%$ & $0 \%$ & \\
\hline & Skin & $0 \%$ & $12.5 \%$ & \\
\hline & Eye & $5.26 \%$ & $12.5 \%$ & \\
\hline \multicolumn{2}{|c|}{$\%$ Isolated } & $31.58 \%$ & $62.5 \%$ & \multirow{4}{*}{$0.03^{*}$} \\
\hline & $n+I T L N$ & $47.37 \%$ & $0 \%$ & \\
\hline \multicolumn{2}{|c|}{ \% EPTB (excl skin \& eye) } & $42.11 \%$ & $12.5 \%$ & \\
\hline \multicolumn{2}{|c|}{$\%$ Hypersens TB } & $5.26 \%$ & $25 \%$ & \\
\hline \multirow{2}{*}{ Cytology } & $\begin{array}{l}\text { Granulomatous } \\
\text { w/ caseation }\end{array}$ & $52.63 \%$ & $0 \%$ & \multirow[t]{2}{*}{$0.01^{*}$} \\
\hline & $\begin{array}{l}\text { Granulomatous } \\
\text { w/o caseation }\end{array}$ & $26.32 \%$ & $75 \%$ & \\
\hline
\end{tabular}

based on clinical severity, disease phenotype and diagnostic and radiological findings. Host response in the lymph nodes is reflected by both symptom manifestation and disease dissemination, implicating the lymph nodes in a critical role in the natural history of TB infection. Finally, a subclinical phenotype was identified, suggesting an early stage of disease progression in TB.

\section{P187 THE USE OF MOXIFLOXACIN FOR THE TREATMENT OF OPHTHALMIC TUBERCULOSIS}

${ }^{1} \mathrm{JL}$ Potter, ${ }^{2} \mathrm{R}$ Agrawal, ${ }^{1} \mathrm{C}$ Barraclough, ${ }^{2} \mathrm{~F}$ Rahman, ${ }^{1} \mathrm{H}$ Kunst, ${ }^{2} \mathrm{M}$ Westcott. ${ }^{1}$ Barts Health NHS Trust, London, UK; ${ }^{2}$ Moorfields Eye Hospital NHS Foundation Trust, London, UK

\subsection{6/thoraxjnl-2014-206260.316}

Background The number of patients we are treating for ophthalmic tuberculosis (TB) have increased year on year, from two in 2009 to twenty in 2013. A recent global review of the strategies used in the diagnosis and treatment of ophthalmic TB showed a wide disparity of diagnostic and treatment strategies. We present a review of our current practice and justification for out treatment regimens.

Methods We identified all the cases in our hospital treated for ophthalmic TB between 2009 and 2013. Age, gender, ophthalmic examination findings, blood tests, treatment regimens, including durations and outcomes, and adverse drug reactions were collected and analysed.

Results A total of 60 cases were identified. Mean age was 45.0 +/- 14.4 years. $61.7 \%$ were male. The most commonly used regimen was 2 months rifampicin, isoniazid, pyrazinamide and moxifloxacin followed by 10 months of rifampicin, isoniazid and moxifloxacin. A response to treatment, with no evidence of disease recurrence on cessation of therapy, was seen in $78.3 \%$ of cases. 5\% experienced hepatotoxicity requiring a change in treatment. There was no significant difference in either the success of treatment $(p=0.102)$ or the risk of hepatotoxicity $(p=0.264)$ between regimens with moxifloxacin $(n=43)$ or without it ( $n$ $=17) .32$ patients on moxifloxacin had ECGs of which 6
(18.8\%) newly developed a raised QTc. This resulted in moxifloxacin being stopped during the step-down phase of treatment in two patients. Maximum QTc was never found to be above 500 milliseconds and there were no episodes of documented arrhythmias or syncope.

Conclusions We recommend a treatment regimen including moxifloxacin in place of ethambutol so that any reported visual change is unlikely to be related to treatment, and we propose continuing moxifloxacin beyond the intensive phase, if tolerated, when culture is unavailable. We treat ophthalmic TB for the same duration as central nervous system TB. Our data shows that this is a safe and effective regimen but more evidence is required before recommending definitive guidelines.

\section{P188 INCREASED PULMONARY M. AVIUM-INTRACELLULARE ISOLATES ACCOUNT FOR MUCH OF THE NATIONAL RISE IN NON-TUBERCULOUS MYCOBACTERIA INCIDENCE, 2007-2012}

${ }^{1} \mathrm{NM}$ Shah, ${ }^{1} \mathrm{~J}$ Davidson, ${ }^{1} \mathrm{~L}$ Anderson, ${ }^{1} \mathrm{HL}$ Thomas, ${ }^{2} \mathrm{M}$ Lipman, ${ }^{1} \mathrm{I}$ Abubakar. ${ }^{1}$ Public Health England, London, UK; ${ }^{2}$ Royal Free London NHS Foundation Trust, London, UK

\subsection{6/thoraxjnl-2014-206260.317}

Introduction The incidence of nontuberculous mycobacteria (NTM) isolation from humans is increasing worldwide. In England, Wales and Northern Ireland (EW and NI) the reported rate of NTM more than doubled between 1996 and 2006. It is unclear if this trend has continued. We present an updated analysis with national NTM data from 2007 to 2012.

Methods All individuals with culture positive NTM isolates between 2007-2012 reported to Public Health England by the five mycobacterial reference laboratories serving EW and NI, were included. The annual incidence of NTM was calculated based on the year of the first positive NTM isolate from each individual.

Results 21,024 individuals had NTM culture positive samples. Over the study period the incidence rose from $5.57(\mathrm{n}=3126)$ to $7.63(\mathrm{n}=4454)$ per 100,000 population. The majority were male $(57 \%)$ and older $(71 \%>50$ years of age). $77 \%$ of individuals had a pulmonary isolate - and here the incidence increased from 3.97 to 6.05 per 100,000 population between 2007 and 2012. In those with extra-pulmonary samples it remained stable at 1.61 per 100,000 population. In patients

Table 1 indicates the seven most frequently reported organisms; M. avium-intracellulare (MAI) accounted for 35\% of isolates $(75 \%$ in people $>50$ years). $78 \%$ of these were from pulmonary samples. $42 \%$ of $\mathrm{M}$. abscessus isolates were in patients 60 years.

\begin{tabular}{llll}
$\begin{array}{l}\text { Abstract P188 Table } 1 \\
\text { samples 2007-2012 }\end{array}$ & Most common NTM reported from total \\
\hline & $\begin{array}{l}\text { Total number } \\
\text { of samples }\end{array}$ & $\begin{array}{l}\text { 2007 incidence } \\
\text { (per 100,000 }\end{array}$ & $\begin{array}{l}\text { 2012 incidence } \\
\text { (per 100,000 } \\
\text { population) }\end{array}$ \\
Organism & $\mathbf{2 0 0 7 - 1 2}$ & 1.90 & 2.80 \\
\hline M. avium-intracellulare & 7400 & 0.74 & 1.38 \\
M. gordonae & 3373 & 0.60 & 0.9 \\
M. chelonae & 2318 & 0.47 & 0.58 \\
M. fortuitum & 1681 & 0.42 & 0.29 \\
M. kansasii & 1299 & 0.34 & 0.34 \\
M. xenopi & 1065 & 0.26 & 0.38 \\
M. abscessus & 986 & & \\
\hline
\end{tabular}


Conclusion The incidence of NTM has continued to rise since the last national survey. This represents an almost ten-fold increase since 1995. The majority of these are pulmonary isolates (in particular MAI). Possible explanations include greater awareness amongst clinicians leading to increased sampling, improvements in laboratory techniques for speciation or laboratory reporting practices. However, such a large increase most likely reflects a genuine rise in NTM infection in the population. Given this change in culture confirmation, it is imperative that a comprehensive clinical database is set up to provide national monitoring of clinically significant infections, and establish the true burden of disease present in EW and NI.

\section{P189 SHOULD SCREENING FOR CHRONIC VIRAL HEPATITIS IN PATIENTS WITH TUBERCULOSIS BE INTRODUCED TO NICE GUIDELINES?}

JL Potter, C Hyams, M Shaukat, ZO Babiker, VM Macavei, N Jayasekera, H Kunst, GR Foster, VLC White. Barts Health NHS Trust, London, UK

\subsection{6/thoraxjnl-2014-206260.318}

Background Screening for viral hepatitis is not routinely recommended in patients diagnosed with tuberculosis (TB). However there are significant similarities in the global distribution of $\mathrm{TB}$ and hepatitis B (HBV) and C (HCV). It remains unclear whether co-infection with HBV or HCV is a risk factor for hepatotoxicity in patients receiving anti-tuberculous therapy and significant morbidity and mortality is associated with a late diagnosis.

Objectives To determine the prevalence of HBV and HCV infection among new cases of active TB across treatment centres in East London and to assess the adverse drug reactions to antituberculous treatment experienced by this population.

Methods We conducted a retrospective study including all patients diagnosed with active TB during 2013 at two TB clinics in London. Data on demographic characteristics, HBV surface antigen (HBsAg), HCV antibody, human immunodeficiency virus (HIV) and adverse drug reactions were retrospectively analysed.

Results In total, 472 cases of active TB were notified during 2013. The mean age was $37.7(+/-15.3)$ years (range: 5-92). Males accounted for $62.3 \%$ of our cohort. $84.7 \%$ of patients were born outside of the UK with the majority of patients being born in either Bangladesh (16.5\%), India (27.8\%) or Pakistan (15.9\%). Overall, 304 patients were screened for HBV, 302 for $\mathrm{HCV}$, and 447 for HIV. Of those screened, HBsAg was detected in $3.3 \%$, HCV antibody in $2.0 \%$ and HIV in $3.4 \%$. All patients infected with HBV or HCV were foreign born. Hepatotoxicity was defined as an ALT greater than 5 times the upper limit of normal or requiring a change in treatment. There was no significant difference in rates of hepatotoxicity in either in HepBsAg status $(\mathrm{p}=0.371)$, HCV status $(\mathrm{p}=0.597)$ or HIV status $(p=0.413)$ but numbers of HBV and HCV infection were small.

Conclusions The prevalence of $\mathrm{HBV}$ and $\mathrm{HCV}$ was significantly higher in our cohort of TB patients than the background UK prevalence, which is $0.4 \%$ for $\mathrm{HCV}, 0.3 \%$ for $\mathrm{HBV}$ and $0.15 \%$ for HIV. Routine screening for HBV and HCV on an opt-out basis would be justified in our setting given the high proportion of foreign-born patients. Further research into the magnitude of $\mathrm{HBV} / \mathrm{HCV}$ co-infection with active or latent TB, any increased risk in drug-induced hepatotoxicity and the cost-effectiveness of routine screening is needed.

\section{P190} DRUG INDUCED LIVER INJURY IN THE TREATMENT OF TUBERCULOSIS IN A BUSY UK CENTRE

${ }^{1} \mathrm{~S}$ Chitty, ${ }^{1} \mathrm{R}$ Ghani, ${ }^{1} \mathrm{JK}$ Roe, ${ }^{1} \mathrm{H}$ Davidson, ${ }^{1} \mathrm{M}$ Routledge, ${ }^{1} \mathrm{~T}$ Edwards, ${ }^{1} \mathrm{C}$ Hateley, ${ }^{2} \mathrm{~S}$ Collin, ${ }^{3}$ A Ritchie, ${ }^{1} \mathrm{~J}$ Dzvova, 'J Buckley, ${ }^{1} \mathrm{RN}$ Davidson, 'L John. 'Northwick Park Hospital, London, UK; ${ }^{2}$ School of Social and Community Science, University of Bristol, Bristol, UK; ${ }^{3}$ Department of Respiratory Medicine, Central Middlesex Hospital, London, UK

\subsection{6/thoraxjnl-2014-206260.319}

Introduction We describe the incidence and management of drug induced liver injury (DILI) in active TB at the largest UK centre, using a nurse-led local protocol derived from 1998 BTS guidelines.

Methods All active TB cases were identified from April 2010 to May 2014. Patients were identified with DILI by following criteria: Type 1 DILI(ALT $>3 \mathrm{x}$ upper limit normal (ULN-55iu/l), Type 2 DILI (ALP $>2 x$ ULN(150 iu/l) and Bilirubin $>21 \mathrm{iu} / \mathrm{l}$ ) or Type 3 DILI (Bilirubin $>40 \mathrm{iu} / \mathrm{l}$ ). Patient demographics, TB treatment (ATT), timing, management and outcomes of DILI were described. Baseline characteristics and ATT doses were matched with controls.

Results 105 individuals with DILI were identified out of 1529 patients with active TB (6.9\%). 81\% were on standard first line therapy (Rifampicin (R), Isoniazid $(\mathrm{H})$, Pyrazinamide $(\mathrm{Z})$ and Ethambutol (E)). 7.8\% were on Moxifloxacin (M) instead of E and $1.9 \%$ were on RHME. Type 1 DILI was most frequent (81\%) with median peak ALT $296 \mathrm{iu} / \mathrm{l}$ (IQR 227-505). Median time from treatment start to onset of DILI was 12.5 days (7-30). Symptoms at presentation included nausea/vomiting (54\%), abdominal pain (18\%) and jaundice (12.4\%). 45.7\% patients had all medication stopped, $7.6 \%$ continued ethambutol with amikacin (A), $26.7 \%$ continued all medication, $6.7 \%$ stopped Z only, 3.8\% substituted Z for a quinolone. Median time from stopping to reintroduction was 10 days (6-17). Of 66 reintroduction patients, regimens included $\mathrm{H}>\mathrm{R}>\mathrm{E}(45 \%)$, $\mathrm{H}>\mathrm{R}>\mathrm{E}>\mathrm{M}(31 \%)$ and $\mathrm{R}>\mathrm{E}>\mathrm{M}(15 \%)$. Median time from reintroduction to full treatment restart was 14 days (12-18). $81 \%$ of patients were uneventfully reintroduced, $5 \%$ suffered a 2 nd DILI. 32\% patients required hospital admission and 4(3.8\%) died.

DILI cases were matched to 200 controls. Cases more likely $(\mathrm{P}<0.05)$ to; be HIV positive, have quinolones in initial regimen and lower body weight. Quinolone use gave an adjusted hazard ratio $5.41(2.96,9.91)$.

Conclusion DILI remains the most important toxicity of ATT and usually occurs during the first month. The BTS guideline provides a useful template for the diagnosis and management of DILI which may be largely nurse led and ambulatory. Most patients are successfully reintroduced without pyrazinamide. HIV status, body weight and quinolone use are risk factors.

\section{P191 WITH A LOW INCIDENCE OF DRUG-INDUCED HEPATITIS, SHOULD WE BE OFFERING LATENT TB TREATMENT TO MORE PATIENTS OVER THE AGE OF 35?}

P Howlett, N Lungu, W Owen, R Breen, L Baker. Guys and St Thomas' Hospital, London, UK

\subsection{6/thoraxjnl-2014-206260.320}

Introduction NICE guidelines recommend patients $>35$ yrs at risk of tuberculosis (TB) on screening, but without active disease, should not be offered latent TB infection (LTBI) treatment unless a healthcare worker, or HIV positive. This is based on perceived 